\title{
Constraint-induced movement therapy for recovery of upper-limb function following traumatic brain injury
}

\author{
Sharon E. Shaw, DrPH, PT; ${ }^{1 *}$ David M. Morris, PhD, PT; ${ }^{1}$ Gitendra Uswatte, PhD; ${ }^{2}$ Staci McKay, BS; ${ }^{1}$ \\ Jay M. Meythaler, MD; Edward Taub, PhD; ${ }^{2,4}$ \\ Departments of ${ }^{1}$ Physical Therapy, ${ }^{2}$ Psychology, and ${ }^{3}$ Physical Medicine and Rehabilitation, University of Alabama \\ at Birmingham, Birmingham, AL; ${ }^{4}$ Department of Veterans Affairs (VA), Research Service, Birmingham VA Medical \\ Center, Birmingham, $A L$
}

\begin{abstract}
A volunteer sample of 22 participants with chronic traumatic brain injury (TBI) (onset $>1$ year) and relative hemiplegia that revealed moderate disability in the more-affected upper limb (UL) participated. Constraint-induced (CI) movement therapy (CI therapy) was employed for a 2-week period; treatments included massed practice, shaping of the moreaffected UL, behavioral contracts, and other behavioral techniques for affecting transfer to a real-world setting. We used the Wolf Motor Function Test, the Fugl-Meyer Motor Performance Assessment, and the Motor Activity Log to measure outcomes. All outcome measures improved significantly as a result of the intervention. More-adherent participants had more improvement compared with less-adherent participants. These preliminary results suggest that CI therapy may be effective for improving UL motor function following chronic TBI.
\end{abstract}

Key words: behavioral therapy, constraint-induced movement therapy, functional abilities, massed practice, motor function, motor impairment, neurological injury, physical rehabilitation, training, traumatic brain injury.

\section{INTRODUCTION}

Traumatic brain injury (TBI) is a highly prevalent problem that results in very large costs to both individuals and society [1]. More than 500,000 people are hospitalized for TBI each year. Of these, approximately 70,000 develop intellectual, behavioral, and/or physical disabilities that prevent their return to an independent lifestyle. More than one-third (39\%) of persons with TBI are between the ages of 16 and 25 at the time of injury, and nearly twothirds are younger than 36; approximately 78 percent are male [2]. Thus, this population consists predominately of young males with a potential for employment. However, postinjury employment outcomes are disappointing [3-6]. Standardized tests of psychological distress confirm the common clinical impression that family caregivers of persons with TBI feel alienated, isolated, overwhelmed, and mentally preoccupied [6]. This was particularly true for

Abbreviations: $\mathrm{ADL}=$ activities of daily living, $\mathrm{CI}=$ constraint-induced, ES = effect size, FA = functional ability, FM = Fugl-Meyer, ICC $=$ intraclass correlation coefficient, MAL = Motor Activity Log, MCID = minimal clinically important difference, $\mathrm{SD}$ = standard deviation, $\mathrm{TBI}$ = traumatic brain injury, $\mathrm{UL}=$ upper limb, WMFT = Wolf Motor Function Test.

This material was based on work supported by the National Institute on Disability and Rehabilitation Research (grant H133A980010) and the Centers for Disease Control and Prevention (grant R49/CCR 403541-12).

*Address all correspondence to Sharon Shaw, DrPH, PT; Department of Physical Therapy, University of Alabama at Birmingham, RMSB 360, 1530 3rd Avenue S, Birmingham, AL 35294; 205-934-3566; fax: 205-975-7787.

Email:sshaw@uab.edu

DOI: 10.1682/JRRD.2005.06.0094 
spouses of persons with TBI. Improvement in motor function for individuals with TBI may improve their functional independence and subsequently reduce the stress on caregivers. While limited studies have reported on the relationship between intensity of therapy and outcomes in this population, evidence exists that increased therapy intensity is positively associated with better motor outcomes [7-10]. A limited number of case studies have been reported with reduced intensity, modified constraintinduced (CI) movement therapy (CI therapy) for persons recovering from TBI [11].

\section{CONSTRAINT-INDUCED MOVEMENT THERAPY}

CI therapy is a rehabilitation approach that is designed to reduce incapacitating motor deficits of the upper limbs (ULs) in patients after neurological injury and increase their functional independence. To date, this approach has been used most frequently with persons recovering from stroke and has been observed to produce improvements in motor function and actual amount of use of a more-affected UL in the real-world environment [12-19]. In our laboratory, CI therapy in the past has involved training of the more-affected UL 6 hours daily for 10 consecutive weekdays over a 2-week period (i.e., concentrated practice or repetitive training). The target is use of the more-affected UL for 90 percent of waking hours for all days of the treatment period and is achieved by employing one of several methods for restraining or reducing the use of the less-affected UL. Evidence from a number of studies suggests that the size of the cortical representation of a body part in adult monkeys and humans depends on the amount of use of that body part [19-21]. Five recent focal transcranial magnetic stimulation, neuroelectric source imaging, and electroencephalograph readiness-potential studies with humans conducted by four groups of investigators and one intracortical microstimulation study with monkeys indicate that CI therapy produces a substantial change in brain organization and function correlative with its large clinical effect [22-26]. A second mechanism associated with the therapeutic effect of CI therapy is overcoming learned nonuse, which has been described in detail elsewhere [1213,19,21].

We performed this present study to determine the effect of employing standard CI therapy in participants with chronic TBI.

\section{METHODS}

\section{Participants}

Following approval of the protocol by the institutional review board, we recruited potential participants by advertising in TBI-related periodicals and conducting workshops and lectures at conferences and rehabilitation facilities. Preliminary screening was conducted via telephone, and potential candidates were given subsequent structured examinations by a physical therapist and a physiatrist.

Inclusion criteria were (1) chronic TBI (onset at least 1 year prior to participation); (2) relative hemiparesis (i.e., one UL more affected than the other); (3) ability to initiate wrist extension, thumb abduction, and extension of two additional fingers from a resting position of forearm pronation and/or full wrist flexion; (4) ability to transfer safely and independently to and from a toilet; (5) ability to stand from a sitting position and maintain standing for $2 \mathrm{~min}$, with UL support if necessary; and (6) score of 6 or better on the Wechsler Memory Scale-III Logical Memory Test I and II [27], 69 or less on the Trail Making Test Part A, and 179 or less on the Trail Making Test Part B [28]. Exclusion criteria were (1) strokeinduced TBI, (2) score of less than 24 on the Folstein Mini-Mental State Examination [29] and a score of 36 or below on the Token Test of the Mutlilingual Aphasia Examination [30], (3) 19 years of age or less, or (4) clinical judgment of either excessive frailty or excessive pain in any joint of the more-affected UL. Participants were characterized as having motor deficits that ranged from mild-to-moderate to moderate-to-severe.

General demographic information for the 22 participants is provided in Table 1. Mean age at entry in the study was $39.3 \mathrm{yr} \pm 14.4$ standard deviation (SD), with an average TBI chronicity of $8.9 \mathrm{yr} \pm 8.8$ SD. Two additional participants entered into the study and later chose not to participate. At entry, both individuals appeared similar to the other participants.

\section{Outcome Measures}

Treatment effectiveness was determined in two separate domains: optimal motor ability as evoked on request in laboratory motor tests and actual spontaneous use of the more-affected UL in a real-world setting [17-18]. The laboratory-based measures of motor ability were the FuglMeyer (FM) Motor Performance Assessment [31-32] and the Wolf Motor Function Test (WMFT) [12,33-35]. The 
Table 1.

Demographic characteristics for participants with TBI $(n=22)$.

\begin{tabular}{lcc}
\hline \multicolumn{1}{c}{ Characteristics } & Frequency & Percentage \\
\hline Male & 14 & 65.2 \\
African American & 3 & 13 \\
Caucasian & 19 & 87 \\
Hand Dominance Prestroke & & \\
$\quad$ Right & 21 & 95.7 \\
More-Affected Upper Limb & & \\
$\quad$ Right & 10 & 47.8 \\
Mode of TBI & & \\
$\quad$ Motor Vehicle Accident & 17 & 77 \\
$\quad$ Gunshot Wound & 1 & $<1$ \\
$\quad$ Fall & 1 & $<1$ \\
$\quad$ Assault & 2 & $<1$ \\
Initial Level of Motor Impairment & & \\
$\quad$ Mild-to-Moderate (Grade 2) & 13 & 59.1 \\
$\quad \begin{array}{l}\text { Moderate (Grade 3) } \\
\quad \text { Moderate-to-Severe (Grade 4) }\end{array}$ & 4 & 18.2 \\
*More-affected upper limb. & 5 & 22.7 \\
TBI = traumatic brain injury. & & \\
\hline \hline
\end{tabular}

measure of real-world use was the Motor Activity Log (MAL) [12,17-18,36]. The FM Motor Performance Assessment is an impairment-based laboratory test of motor ability that examines sensation, range of motion, pain, and quality of movement during a series of increasingly difficult motor tasks [31-32]. The FM Motor Performance Assessment has been used as a clinical and research tool for evaluating changes in motor impairment following stroke [31]. It has also been used to measure motor capabilities of persons recovering from other neurologic disorders, including TBI [37-39]. We used the UL portion of the FM Motor Performance Assessment (total UL score ranged from 0 to 66 , based on a score of 0,1 , or 2 for each of the 33 test items). We administered the FM Motor Performance Assessment at pretreatment, posttreatment, and 2-year follow-up.

The WMFT is a laboratory-based performance test that was developed by Wolf and modified by Taub et al. for quantification of optimal motor ability as requested of persons recovering from stroke and TBI [12,33-35]. The version of the WMFT that we employed consists of 17 motor tasks: 15 are timed and 2 involve measures of strength. The 15 timed tasks were filmed and later rated for functional ability (FA) with an 8-point rating scale ( $0-7)$ by raters who were blinded to treatment status. We tested both ULs. The less-affected UL was tested first, which has the advantage of enhancing the participant's understanding of the task instructions. The test requires standard participant testing positions; it provides an index of the upper limit of motor ability for that participant at that time. Like the FM Motor Performance Assessment, the WMFT was administered at pretreatment, posttreatment, and 2-year follow-up. Only the FA score is reported because it is more reliable than the performance time score (median of item scores) [34].

The MAL is a structured interview in which respondents are asked to rate how much (Amount of Use scale) and how well (Quality of Movement scale) they use their more-affected UL for 30 activities of daily living (ADL) outside the laboratory [12,17-18]. The MAL was administered independently to participants and one of their caregivers, if one was available. The test items include such activities as brushing teeth, buttoning a shirt or blouse, and eating with a fork or spoon. The entire MAL was administered the day before and the day after the intervention and at 1-month and 2-year follow-up. In addition, the Quality of Movement scale was administered to the participants daily during the treatment period. Only the Quality of Movement scale, referred to from here forward as the Arm Use scale, is reported because the Amount of Use scale is highly correlated with the Arm Use scale (i.e., redundant) and less reliable [17-18,40]

\section{Interventions}

During the 2-week CI therapy intervention, participants spent $6 \mathrm{~h}$ each weekday in the laboratory engaging in repetitive or massed practice, shaping [41-42], or taskpractice procedures. The shaping procedure involved (1) selecting tasks that address the motor deficits of the individual participant; (2) encouraging the patient to keep trying to improve the speed and/or quality of performance on the next trial; (3) helping the participant to carry out parts of a movement sequence if they were incapable of completing the movement independently; and (4) providing explicit verbal feedback, quantitative information, and verbal reward for small improvements in task performance. Modeling, prompting, and cueing of task performance were also employed. In addition, participants wore a protective safety mitt (Sammons Preston Rolyan, Bolingbrook, Illinois) on their less-affected UL. This greatly reduced their ability to use the less-affected UL and thereby induced increased use of the more-affected UL. While they were supervised by research personnel in the laboratory, participants wore the protective safety mitt on their less-affected UL 90 percent of the time. When the participants were outside the laboratory, we encouraged 
mitt wearing to increase use of the more-affected UL with behavioral techniques, such as a behavioral contract and a home diary. The behavioral contract was formulated on the first day of treatment in conference with the participant; it specified the activities the participant should perform with the more-affected UL alone and the activities for which the mitt could be removed (i.e., when water was used, toileting, some dressing tasks, use of an assistive device for ambulation). Safety was an overriding consideration in determining when the mitt could be removed. The contract was agreed upon jointly and signed by the participant, the therapist, and a witness for emphasis of the character of the document as a contract. The participant filled out the home diary and detailed the ways they used the more-affected UL since leaving the laboratory and when and for how long they did not wear the mitt. The home diary was reviewed with the interventionist at the beginning of each treatment day, and problem-solving was engaged in for determination of how the participant could perform activities so that the mitt need not be removed as often, and also how more of the activities specified in the behavioral contract could be performed. When available, caregivers were enlisted to assist participants by serving as a "second arm" for tasks that were bilateral in nature or too difficult for the participant to do with the more-affected UL alone. During the last few days of the intervention, an individualized, written home program was established for each participant to complete after the end of treatment. Commonplace tasks were chosen for improving the participants' most significant movement deficits. Such tasks included participants pouring dried beans from a glass to receptacles of progressively smaller sizes, reaching out to place rings on the posts of a ring toss game at progressively longer distances, picking up cotton balls and placing them into a receptacle, and stacking Styrofoam cups. The tasks involved use of equipment typically found in the home or easily found and purchased. The home program consisted of approximately 10 tasks, and participants were encouraged to perform 2 to 3 tasks during a 30 min practice period each day.

\section{Data Analysis}

The effect of treatment on real-world use of the moreaffected UL as measured by the MAL was evaluated with the use of repeated-measures analysis of variance $(\alpha=$ 0.05 , two-tails) with Huynh-Feldt correction for violation of the sphericity assumption. This omnibus test, which assessed changes across all assessment occasions (pretreatment, posttreatment, 1-month follow-up, and 2-year follow-up), was followed by Tukey's tests. These permitted evaluation of differences between particular testing occasions [43]. We evaluated the effect of treatment on motor ability exhibited in the laboratory when motor performance was requested (FM Motor Performance Assessment, WMFT) by comparing pretreatment with posttreatment scores with the use of paired $t$-tests $(\alpha=0.05$, two-tails). Inflation of Type I error because of multiple tests within this domain was controlled with a modification of the Bonferroni correction developed by Benjamini and Hochberg [44]. The magnitude of the treatment gains were represented with a repeated-measures index (i.e., $d$ ) of effect size (ES) [45]. In the meta-analysis literature, $d$ values of 0.14 are considered small, 0.35 are deemed moderate, and 0.57 and above are judged large [45]. In addition, two post hoc analyses were conducted with respect to differences in treatment outcomes among subgroups formed on the basis of amount of (1) adherence to the protocol and (2) initial motor ability.

The main measure of adherence for which quantitative data were available was the percentage of waking hours the mitt was worn. Adherence to mitt wearing in the laboratory was recorded in a log kept by research personnel. Adherence outside the laboratory was tracked with a diary kept by the participant or caregiver. Each day's entries were reviewed with the therapist on the morning of the next day. For four participants, an objective record of adherence outside the laboratory was obtained with a sensor (conductance circuit) and timing device that were sewn into the mitt. Agreement between the self-report (i.e., diary) and objective (i.e., compliance device) measures was calculated daily for each of these four participants with an intraclass correlation coefficient (ICC) Type 2,1 [45]. The agreement between the selfreport and objective measures was excellent (median ICC Type $\left._{2,1}\right)=0.97$, range $=0.89-0.97$ ), which suggests that the diaries provided an accurate measure of adherence outside the laboratory. All participants were subdivided into two groups based on a median split for adherence outside the laboratory (more adherence $>57 \%$; less adherence $<57 \%$ ). The real-world treatment outcomes of these subgroups were compared with analysis of covariance ( $\alpha=0.05$, one-tail). Difference in initial moreaffected UL use among the groups was controlled for by use of the pretreatment MAL score as a covariate. We used the level of participants' initial motor deficit to categorize the participant sample into 3 grades or levels of motor impairment. In past studies, we had described 
these groups as quartiles (Grade 2 = Quartile 1, Grade 3 = Quartile 2, Grade $4=$ Quartile 3). The reason for the change is that participants are not distributed evenly in the quartile description system. Grade 2 (mild-to-moderate impairment) includes individuals with the ability to carry out at least $20^{\circ}$ extension at the wrist and $10^{\circ}$ at each of the fingers from a resting position of forearm pronation and/or full wrist flexion. Grade 3 (moderate impairment) participants did not meet the Grade 2 criteria but had the ability to move into $10^{\circ}$ of wrist extension, $10^{\circ}$ of abduction of the thumb, and $10^{\circ}$ of extension of at least two other digits from the same starting position. Grade 4 (moderate-to-severe impairment) participants did not meet Grade 3 criteria but had the ability to initiate wrist extension, thumb abduction, and extension of at least 2 additional fingers. The number of participants in this study who met the initial motor criteria for Grades 2, 3 , and 4 were 13,4 , and 5, respectively. Grade 1 participants, who have minimal motor impairment, were excluded from this study as were Grade 5 participants, who have essentially plegic hands. The amount of motor function gain as a result of treatment at each grade was compared with the use of mean, SD, and ES statistics. Only descriptive statistics were used for this purpose because the small number of participants in the lower- functioning grades limited the power of any inferential statistics (Table 1).

\section{RESULTS}

\section{Real-World Upper-Limb Use}

A significant improvement was noted in real-world more-affected UL use across all posttreatment testing occasions as measured by the MAL, $F_{(2.8,49.7)}=54.1, p<$ 0.001 (Table 2). Subjects reported very large gains (mean change $=1.6, \mathrm{SD}=0.8$, ES $=2.1$ ) from pretreatment to posttreatment, $Q_{(54)}=12.8, p<0.05$, with no decrement in UL use at 1-month follow-up (Table 2). At 2-year follow-up, there was a 21 percent decline in moreaffected UL use compared with posttreatment, $Q_{(54)}=$ 4.3, $p<0.05$ (Table 2).

Participants' reports were confirmed by the caregivers' MAL scores. Caregivers observed large improvement in participant real-world more-affected UL use (mean change $=1.2$, SD $=0.8$, ES $=1.5$ ) from pretreatment to posttreatment, $t_{(7)}=4.5,(p<0.01)$. The number of caregivers available at the 1-month and 2-year followup was too small for meaningful analysis of the data. Caregiver data at posttreatment were available in only eight cases, since many participants lived independently

Table 2.

Mean \pm standard deviation for Motor Activity Log Arm Use scale scores and effect sizes for treatment changes.

\begin{tabular}{|c|c|c|c|c|c|c|c|}
\hline Impairment Grade* & Pretreatment & Posttreatment & $\begin{array}{c}\text { 1-Month } \\
\text { Follow-Up }\end{array}$ & $\begin{array}{c}\text { 2-Year } \\
\text { Follow-Up }\end{array}$ & $\Delta$ Posttreatment & $\begin{array}{l}\Delta \text { 1-Month } \\
\text { Follow-Up }\end{array}$ & $\begin{array}{c}\Delta \text { 2-Year } \\
\text { Follow-Up }\end{array}$ \\
\hline $\begin{array}{l}\text { All Participants } \\
(N=22)\end{array}$ & $0.9 \pm 0.6$ & $2.4 \pm 1.0$ & $2.5 \pm 1.0$ & $1.9 \pm 0.9$ & $1.6 \pm 0.8^{\S}$ & $1.7 \pm 0.8 \S$ & $1.0 \pm 0.8 \S$ \\
\hline Effect Size & - & - & - & - & 2.1 & 2.1 & 1.3 \\
\hline Grade $2(n=13)$ & $1.2 \pm 0.6$ & $2.9 \pm 0.9$ & $3.0 \pm 0.9$ & $2.1 \pm 0.9$ & $1.7 \pm 0.8$ & $1.8 \pm 0.8$ & $1.0 \pm 0.9$ \\
\hline Effect Size & - & - & - & - & 2.2 & 2.4 & 1.1 \\
\hline Grade $3(n=4)$ & $0.7 \pm 0.4$ & $2.4 \pm 0.7$ & $2.8 \pm 0.5$ & $2.1 \pm 0.7$ & $1.7 \pm 0.7$ & $2.1 \pm 0.6$ & $1.4 \pm 0.7$ \\
\hline Effect Size & - & - & - & 一 & 2.4 & 3.7 & 2.0 \\
\hline Grade $4(n=5)$ & $0.3 \pm 0.2$ & $1.4 \pm 0.8$ & $1.3 \pm 0.8$ & $1.0 \pm 0.6$ & $1.1 \pm 0.8$ & $1.0 \pm 0.9$ & $0.7 \pm 0.5$ \\
\hline Effect Size & - & - & - & - & 1.4 & 1.2 & 1.4 \\
\hline \multicolumn{8}{|c|}{$\begin{array}{l}\text { Note: Significance levels not indicated for each impairment grade because inadequate power existed for evaluation of changes within smaller subgroups (i.e. } \\
\text { Grades } 3 \text { and } 4 \text { ). } \\
{ }^{*} \text { Grade } 2,3 \text {, and } 4 \text { participants had mild-to-moderate, moderate, and moderate-to-severe motor impairment, respectively, of more-affected upper limb prior to treatment } \\
{ }^{\dagger} \text { Data collected from } 95 \% \text { of participants. } \\
{ }^{\ddagger} \text { Data collected from } 87 \% \text { of participants. } \\
\$_{p}<0.05 \text {. } \\
{ }^{q} \text { Reported effect sizes are } d \text { (mean change divided by standard deviation). According to meta-analysis literature, small, medium, and large } d \text {-values are } 0.13 \text {, } 0.35 \\
\text { and } 0.57 \text {, respectively. Source: Benjamini Y, Hochberg Y. Controlling the false discovery rate: A practical and powerful approach to multiple testing. J R Stat Soc } \\
\text { B. 1995;57:289-300. }\end{array}$} \\
\hline
\end{tabular}


or had caregivers who did not participate in the study. However, no significant differences in initial motor ability or treatment outcome were noted between subjects with or without caregivers.

\section{Laboratory Motor Function Tests}

Improvement in more-affected UL motor function was also observed on the FM Motor Performance Assessment (Table 3) for movements performed on request in the laboratory. A large change (mean change $=4.2, \mathrm{SD}=3.5$; $\mathrm{ES}=$ 1.4) existed in the total UL score from pretreatment to posttreatment $t_{(20)}=6.3, p<0.001$. Improvements were greatest for movement of the upper arm (mean change $=4.8$, $\mathrm{SD}=3.5, \mathrm{ES}=1.2$ ) and hand and wrist (mean change 2.6, $\mathrm{SD}=2.9$, $\mathrm{ES}=0.7)$. Changes in range of motion, sensation, and pain scores were small $(\mathrm{ES}<0.2)$.

Improvements in more-affected UL motor ability were also evident on the WMFT (Table 3). Participants showed large gains (mean change $=0.4, \mathrm{SD}=0.53$, $\mathrm{ES}=$ $0.7)$ from pretreatment to posttreatment on the FA scale, $t_{(19)}=3.4, p<0.01$.

\section{Relationship of Real-World Treatment Outcome to Level of Adherence}

Based on a median split (57\%) of adherence to mitt wearing outside of the laboratory, less-adherent participants had smaller treatment gains than more-adherent participants. On the MAL, less-adherent participants showed a trend toward a smaller gain (mean $=1.3, \mathrm{SD}=$ 0.98 , ES $=1.3$ ) in real-world treatment outcome than more-adherent subjects (mean $=1.8, \mathrm{SD}=0.46, \mathrm{ES}=$ 3.9, $\left.\left.F_{(1,19)}=2.5\right), p=0.065\right)$. Furthermore, the amount of gain as a result of treatment was strongly correlated with degree of adherence among less-adherent participants $(r=0.68, p<0.05)$, while no association between these parameters was observed among more-adherent participants (Figure 1); this result suggests that there is a threshold above which degree of adherence makes no additional contribution to treatment outcome. It is important to note, however, that although less-adherent subjects displayed smaller real-world motor function gains as a result of treatment than more-adherent subjects, lessadherent subjects still showed significant gains in realworld UL use, $t_{(10)}=4.3, p<0.01$.

\section{Relationship of Treatment Outcome to Initial Motor Ability of More-Affected Upper-Limb}

Participants with moderate impairment (Grade 3) exhibited motor function gains as a result of treatment that were as good as or better than those exhibited by participants with mild-to-moderate impairment (Grade 2), while participants with moderate-to-severe impairment (Grade 4) displayed somewhat smaller real-world gains

Table 3.

Mean \pm standard deviation for laboratory motor ability test scores and effect sizes for treatment changes by impairment level.

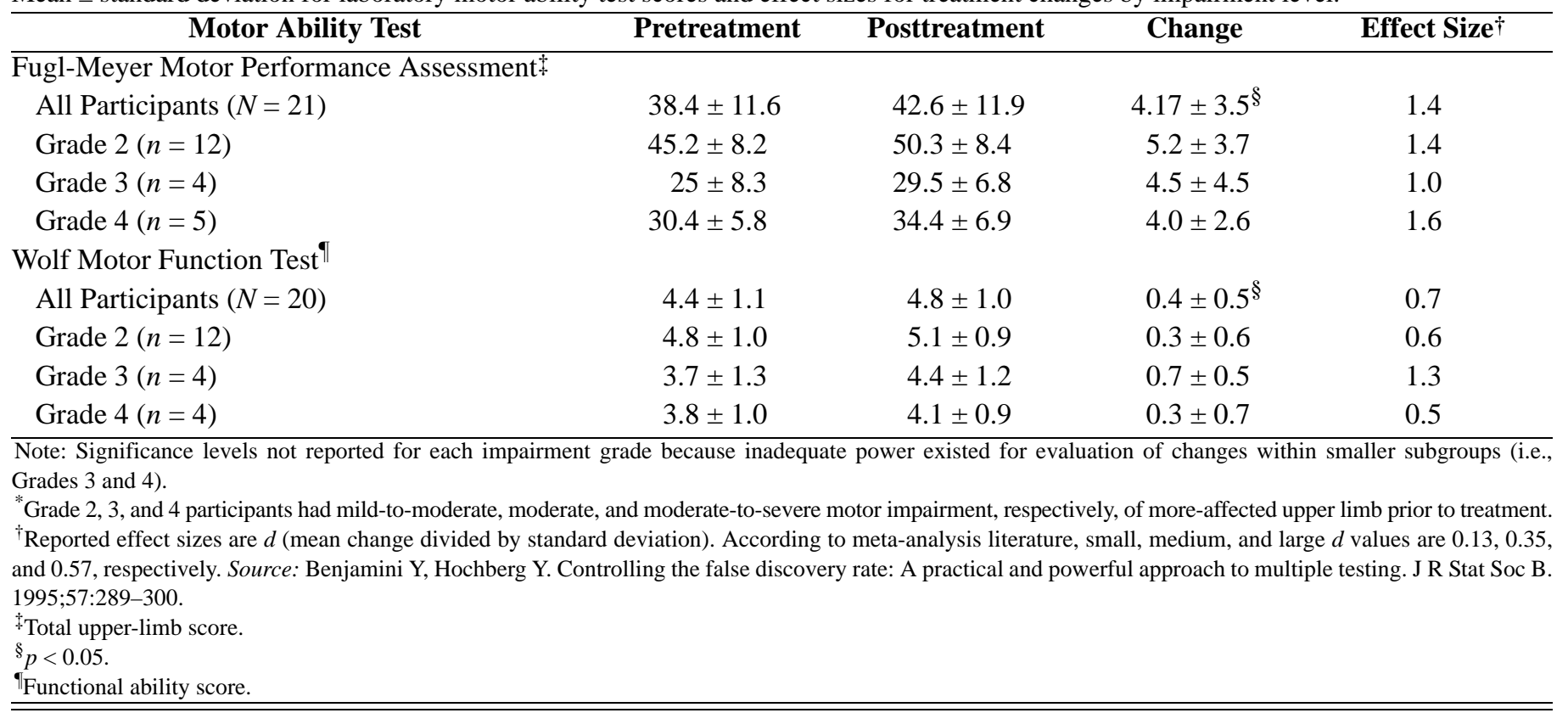




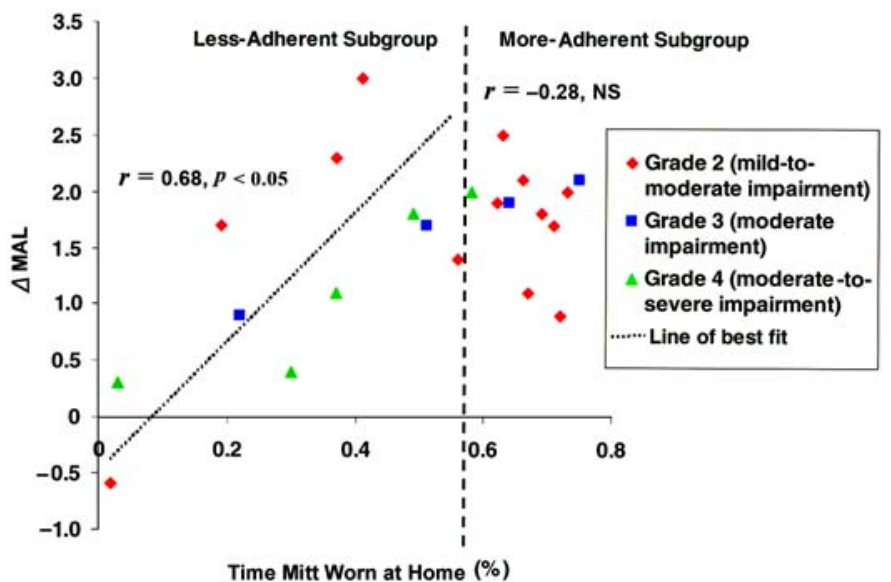

Figure 1.

Scatter plot of participant adherence to mitt wearing on less-affected upper limb (UL) versus gains in Motor Activity Log (MAL) (posttreatment - pretreatment, $\triangle \mathrm{MAL}$ ) for participants with different levels of pretreatment impairment. Study participants were divided into less-adherent and more-adherent subgroups (median split, i.e., $57 \%$ [dashed, bold line]) based on proportion of waking hours that mitt was worn on less-affected UL at home during treatment period. A strong correlation existed between percent adherence and amount of treatment gain among less-adherent participants ( $r=0.68, p<$ 0.05 ), but no correlation existed between these two parameters for more-adherent participants ( $r=-0.28$, not significant [NS]).

than participants with mild-to-moderate (Grade 2) or moderate (grade 3) impairment. For Grade 3 participants, gains on the MAL and FM Motor Performance Assessment were very similar to those for Grade 2 participants, while gains on the WMFT were more than double those for Grade 2 participants (Tables 2 and 3, Figures 2 and 3). For Grade 4 participants, gains on the WMFT and FM Motor Performance Assessment were similar to those for Grade 2 participants, while gains on the MAL were 65 percent of those for Grade 2 participants. Smaller gains on the MAL for Grade 4 relative to Grade 2 and 3 participants cannot, however, be attributed with certainty to level of pretreatment motor impairment per se because a low degree of adherence among Grade 4 participants (4/5 of Grade 4 participants fell into the less-adherent subgroup) might provide an explanation for this pattern.

\section{DISCUSSION}

Study participants were typical of the chronic TBI population in that they were mostly young adult males

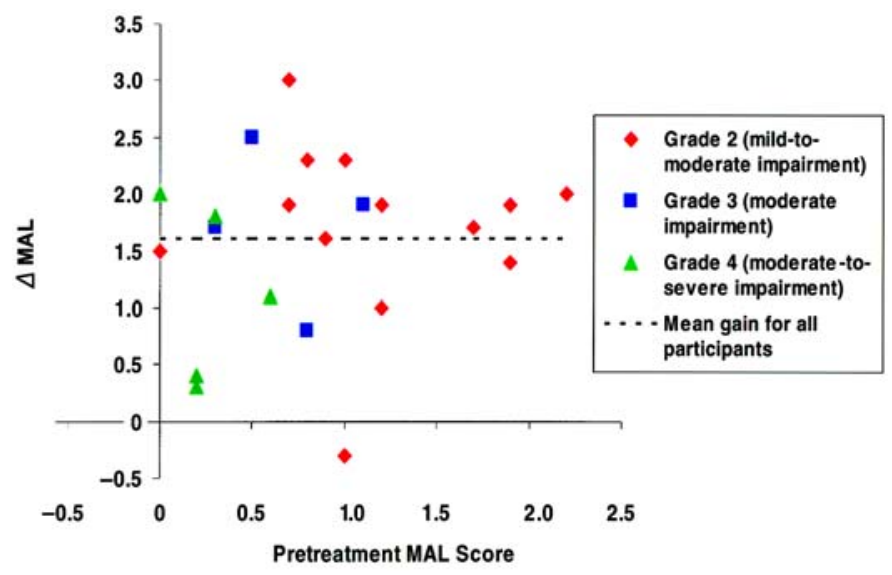

Figure 2.

Scatter plot of pretreatment scores on Motor Activity Log (MAL) vs gains in MAL (posttreatment - pretreatment, $\triangle$ MAL) for participants with different levels of pretreatment impairment. Little to no relationship existed between pretreatment MAL Arm Use scale scores and posttreatment gains on this measure. Posttreatment gains for individual participants were more or less randomly distributed around mean for all participants regardless of individual pretreatment scores. However, participants with moderate-to-severe motor impairments of more-affected upper limb prior to treatment (i.e., Grade 4) showed smaller treatment gains on MAL than participants with less initial motor impairment (i.e., Grades 2 and 3).

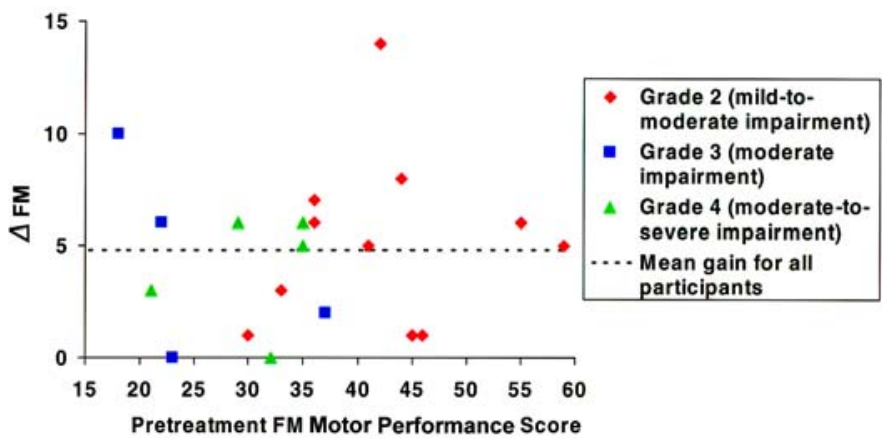

Figure 3.

Scatter plot of Fugl-Meyer (FM) Motor Performance Assessment gains (posttreatment - pretreatment, $\Delta \mathrm{FM}$ ) vs pretreatment scores for participants with different levels of pretreatment impairment. Little or no relationship existed between pretreatment FM Motor Performance Assessment total upper-limb scores and posttreatment changes on this measure or between initial level of motor impairment (more-affected upper limb) and posttreatment gains on FM Motor Performance Assessment.

who possessed mild-to-moderate cognitive deficits. After 2 weeks of CI therapy, they demonstrated improvements in all outcome measures; the average improvement by all 
participants on the MAL, which captures how well and how much individuals actually use their more-affected UL for accomplishing ADL outside of the laboratory, was very large (posttreatment ES $=2.1$ ) by the standards of the meta-analysis literature and more than four times greater than what has been identified by another laboratory as a minimal clinically important difference (MCID) $(\mathrm{ES}=0.05)$ [36]. Furthermore, participants at each grade of more-affected UL motor impairment showed large real-world effects: Grade 2 participants MAL ES $=2.2$, Grade 3 participants MAL ES $=2.4$, and Grade 4 participants MAL ES = 1.4. Not surprisingly, since participants with greater motor impairment began treatment with less use of their more-affected UL than participants with smaller motor impairment and thus had lower scores on the MAL (e.g., pretreatment MAL means for Grades 4 and 2 were 0.3 and 1.2, respectively), their posttreatment level of more-affected UL use was also less than that of higher functioning participants (e.g., posttreatment MAL means for Grades 4 and 2 were 1.4 and 2.9, respectively). Nevertheless, even the most impaired participants showed a clinically meaningful improvement; mean gains on the MAL for Grade 4 participants were more than twice the MCID. Other ways to evaluate the meaningfulness of the results are that Grade 4 participants scored $>2$ (i.e., more-affected UL was of some use for accomplishing the task) on 15 and 47 percent of MAL items before and after treatment, respectively, and that after treatment these participants, who had moderate-tosevere motor deficits, moved into the upper range of UL use found in participants with only mild-to-moderate motor deficits (i.e., Grade 2).

Those participants with TBI who were more-adherent in mitt wearing experienced treatment outcomes equivalent to participants with stroke in previous studies [12,1718]. More-adherent participants with TBI who received an adequate "dose" of CI therapy achieved a real-world treatment outcome (MAL mean gain $=1.8, \mathrm{SD}=0.46$, $\mathrm{ES}=3.9$ ) as least as good as that of participants recovering from strokes treated in this laboratory who had the same grade of initial motor impairment and received similar treatment $(\mathrm{MAL}$ mean gain $=1.8, \mathrm{SD}=0.6, \mathrm{ES}=3.3$ ) [19]. The values and statistics reported here for stroke survivors are a weighted average of those for Grade $2(n=$ $21)$, Grade $3(n=10)$, and Grade $4(n=13)$ participants treated in this laboratory with a protocol similar to the one we used here for participants with TBI [19]. The weights assigned to the values for the groups with stroke were in proportion to the number of participants from each grade of motor impairment in the more-adherent subgroup with TBI.

Adherence with wearing the mitt on the less-affected UL for participants with stroke (mean overall adherence = $76 \%$, SD $=9.5$; weighted-average) was similar to that for more-adherent participants with TBI (mean overall adherence $=73 \%$, SD $=5$ ). Lower adherence with mitt wearing in the clinic and home appears to be associated with reduced real-world treatment outcomes. The data, however, do not support a causal relationship between adherence with wearing the mitt and treatment outcome per se. Lack of compliance with mitt wearing may have been associated with a variety of other factors that were more directly responsible for the diminished treatment effect. Examples of such problems include lesser motivation to improve motor function, low frustration tolerance, poor memory, excessive fatigue, distractibility, and emotional outbursts.

However, all participants, including those who were less-adherent, showed improved motor function following CI therapy. This suggests that even those participants with cognitive/behavioral problems might benefit from this intervention, especially if a program of behavior management is employed to increase cooperation with the requirements of the therapy. Potential solutions to increase adherence both in the home and in the clinic might include structuring participants' schedules outside the laboratory in a more focused and organized manner and enlisting greater caregiver support by encouraging and educating both participants and caregivers. Cognitive training and behavioral management strategies to address this problem could also be incorporated into the CI therapy protocol in future studies.

Questions that remain to be explored are whether the positive outcomes with these 22 participants would be obtained with a larger sample and in other laboratories. To the extent that similar mechanisms are responsible for the gains observed after CI therapy for both the participants with TBI in this study and the stroke survivors in previous CI therapy studies, the results from this study are unlikely a result of nonspecific factors such as therapist attention and test order. CI therapy for individuals with chronic stroke has been shown to be efficacious in controlled studies [19] and to produce large changes in cortical representations of more-affected UL function [20]. Furthermore, the participants were more than 1 year post-TBI prior to enrollment and by extensive clinical 
experience and traditional belief, additional improvement in UL motor function either from spontaneous recovery or administration of a rehabilitation therapy would have been unlikely. Nevertheless, since no control subjects were involved in this study, a randomized, placebocontrolled trial to conclusively rule out nonspecific factors as alternate explanations for the findings would be valuable. Another important issue is determining which strategies are helpful for maximizing adherence to the CI therapy protocol.

\section{CONCLUSIONS}

These preliminary findings suggest that CI therapy may be effective for improving UL motor function following chronic TBI. Protocol adherence would seem to be an important factor in determining treatment outcome.

\section{ACKNOWLEDGMENTS}

We would like to thank the following colleagues for their help and advice at difficult stages of the work: Jean E. Crago, Victor Mark, Francilla Allen, Danna Kay King, Camille Bryson, Sonya Pearson, Christy Bussey, Sherry Yakley, Thomas Novack and, in particular, Stephanie Abernathy.

\section{REFERENCES}

1. Fulk GD, Geller A. Traumatic brain injury. In: O’Sullivan SB, Schmitz TJ, editors. Physical rehabilitation: Assessment and treatment. Philadelphia (PA): FA Davis; 2001. p. 783-819.

2. Gordon WA, Mann N, Willer B. Demographic and social characteristics of the traumatic brain injury model system database. J Head Trauma Rehabil. 1993;8(2):26-33.

3. Yasuda S, Wehman P, Targett P, Cifu D, West M. Return to work for persons with traumatic brain injury. Am J Phys Med Rehabil. 2001;80(11):852-64.

4. Brooks N, McKinlay W, Symington C, Beattie A, Campsie L. Return to work within the first seven years of severe head injury. Brain Inj. 1987;1(1):5-19.

5. Ben-Yishay Y, Silver SM, Piasetsky E, Rattock J. Relationship between employability and vocational outcome after intensive holistic cognitive rehabilitation. J Head Trauma Rehabil. 1987;2(1):35-48.
6. Gervasio AH, Kreutzer HS. Kinship and family members' psychological distress after traumatic brain injury: A large sample study. J Head Trauma Rehabil. 1997;12:14-26.

7. Blackerby WF. Intensity of rehabilitation and length of stay. Brain Inj. 1990;4(2):167-73.

8. Aronow HU. Rehabilitation effectiveness with severe brain injury: Translating research into policy. J Head Trauma Rehabil. 1987;2:24-36.

9. Heinemann AW, Hamilton B, Linacre JM, Wright BD, Granger C. Functional status and therapeutic intensity during inpatient rehabilitation. Am J Phys Med Rehabil. 1995; 74(4):315-26.

10. Cifu DX, Kreutzer JS, Kolakowsky-Hayner SA, Marwitz $\mathrm{JH}$, Englander J. The relationship between therapy intensity and rehabilitative outcomes after traumatic brain injury: A multicenter analysis. Arch Phys Med Rehabil. 2003;84(10):1441-48.

11. Page S, Levine P. Forced use after TBI: Promoting plasticity and function through practice. Brain Inj. 2003;17(8): 675-84.

12. Taub E, Miller NE, Novack TA, Cook EW 3rd, Fleming WC, Nepomuceno CS, Connell JS, Crago JE. Technique to improve chronic motor deficit after stroke. Arch Phys Med Rehabil. 1993;74(4):347-54.

13. Morris D, Crago J, DeLuca S, Pidikiti R, Taub E. Constraint-induced (CI) movement therapy for motor recovery after stroke. NeuroRehabilitation. 1997;9:29-43.

14. Taub E, Crago JE, Uswatte G. Constraint-induced movement therapy: A new approach to treatment in physical rehabilitation. Rehabil Psychol. 1998;43(2):152-70.

15. Miltner WH, Bauder H, Sommer M, Dettmers C, Taub E. Effects of constraint-induced movement therapy on patients with chronic motor deficits after stroke: A replication. Stroke. 1999;30(3):586-92.

16. Kunkel A, Kopp B, Muller G, Villringer K, Villringer A, Taub E, Flor H. Constraint-induced movement therapy for motor recovery in chronic stroke patients. Arch Phys Med Rehabil. 1999;80(6):624-28.

17. Taub E, Uswatte G. Constraint-induced (CI) movement therapy based on behavioral neuroscience. In: Frank RG, Elliot TR, editors. Handbook of rehabilitation psychology. Washington (DC): American Psychological Association; 2000. p. 475-96.

18. Uswatte G, Taub E. Implications of the learned nonuse formulation for measuring rehabilitation outcomes: Lessons from Constraint-Induced Movement therapy. Rehabil Psychol. 2005;50:34-42.

19. Taub E, Uswatte G. Constraint-induced movement therapy: Bridging from the primate laboratory to the stroke rehabilitation laboratory. J Rehabil Med. 2003;35(Suppl 41):S34-40. 
20. Taub E, Uswatte G, Morris DM. Improved motor recovery after stroke and massive cortical reorganization following constraint-induced movement therapy. Phys Med Rehabil Clin N Am. 2003;14(Suppl 1):S77-91, ix.

21. Taub E. Somatosensory deafferentation research with monkeys: Implications for rehabilitation medicine. In: Ince LP, editor. Behavioral psychology in rehabilitation medicine: Clinical applications. New York (NY): Williams \& Wilkins; 1980. p. 371-401.

22. Liepert J, Bauder H, Wolfgang HR, Miltner WH, Taub E, Weiller C. Treatment-induced cortical reorganization after stroke in humans. Stroke. 2000;31(6):1210-16.

23. Kopp B, Kunkel A, Muehlnickel W, Villringer K, Taub E, Flor H. Plasticity in the motor system related to therapyinduced improvement of movement after stroke. Neuroreport. 1999;10(4):807-10.

24. Bauder H, Sommer M, Taub E, Miltner WH. Effects of CI therapy on movement-related brain potentials [abstract]. Psychophysiology. 1999;36(Suppl 1):S31.

25. Levy CE, Nichols DS, Schmalbrock PM, Keller P, Chakeres DW. Functional MRI evidence of cortical reorganization in upper-limb stroke hemiplegia treated with constraintinduced movement therapy. Am J Phys Med Rehabil. 2001;80(1):4-12.

26. Nudo RJ, Wise BM, SiFuentes F, Milliken GW. Neural substrates for the effects of rehabilitative training on motor recovery after ischemic infarct. Science. 1996;272(5269): 1791-94.

27. Wechsler D. Wechsler memory scale III. Administration and scoring manual. San Antonio (TX): Psychological Corporation; 1997.

28. Army individual test battery: Manual of directions and scoring. Washington (DC): War Department, Adjutant General's Office; 1944.

29. Bleecker M, Bolla-Wilson K, Kawas C, Agnew J. Agespecific norms for the Mini-Mental State Exam. Neurology. 1988;38(10):1565-68.

30. Benton AL. Multilingual aphasia examination: Manual of instructions. Iowa City (IA): Department of Neurology and Psychology, University of Iowa; 1983.

31. Fugl-Meyer AR, Jaasko L, Leyman I, Olsson S, Steglind S. The post-stroke hemiplegic patient: 1 . A method for evaluation of physical performance. Scand J Rehabil Med. 1975; 7(1):13-31.

32. Gladstone DJ, Danells CJ, Black SE. The Fugl-Meyer assessment of motor recovery after stroke: A critical review of its measurement properties. Neurorehabil Neural Repair. 2002;16(3):232-40.
33. Wolf SL, Lecraw DE, Barton LA, Jann BB. Forced use of hemiplegic upper extremities to reverse the effect of learned nonuse among chronic stroke and head-injured patients. Exp Neurol. 1989;104(2):125-32.

34. Morris DM, Uswatte G, Crago JE, Cook EW 3rd, Taub E. The reliability of the Wolf Motor Function Test for assessing upper extremity function after stroke. Arch Phys Med Rehabil. 2001;82(6):750-55.

35. Wolf SL, Catlin PA, Ellis M, Archer AL, Morgan B, Piacentino A. Assessing the Wolf Motor Function Test as an outcome measure for research in patients after stroke. Stroke. 2001;32(7):1635-39.

36. Van der Lee JH, Beckerman H, Knol DL, deVet HC, Bouter LM. Clinimetric properties of the motor activity log for the assessment of arm use in hemiparetic patients. Stroke. 2004;35(6):1410-14.

37. Van Wijck FM, Pandyan AD, Johnson GR, Barnes MP. Assessing motor deficits in neurological rehabilitation: Patterns of instrument usage. Neurorehabil Neural Repair. 2001; 15(1):23-30.

38. McCrea PH, Eng JJ, Hodgson AJ. Biomechanics of reaching: Clinical implications for individuals with acquired brain injury. Disabil Rehabil. 2002;24(10):534-41.

39. Alon G, McBride K. Persons with C5 or C6 tetraplegia achieve selected functional gains using a neuroprosthesis. Arch Phys Med Rehabil. 2003;84(1):119-24.

40. Uswatte G, Foo WL, Olmstead H, Lopez K, Holand A, Simms LB. Ambulatory monitoring of arm movement using accelerometry: An objective measure of upperextremity rehabilitation in persons with chronic stroke. Arch Phys Med Rehabil. 2005;86(7):1498-1501.

41. Taub E, Crago JE, Burgio LD, Miller NE, Groomes TE, Cook EW 3rd, DeLuca SC. An operant approach to rehabilitation medicine: Overcoming learned nonuse by shaping. J Exp Anal Behav. 1994;61(2):281-93.

42. Morgan WG. The shaping game: A teaching technique. Behav Ther. 1974;5:271-72.

43. Stevens J. Applied multivariate statistics for the social sciences. Mahwah (NJ): Lawrence Erlbaum Associates; 2001. p. 222-25.

44. Benjamini Y, Hochberg Y. Controlling the false discovery rate: A practical and powerful approach to multiple testing. J R Stat Soc B. 1995;57:289-300.

45. Cohen J. Statistical power analysis for the behavioral sciences. New York (NY): Academic Press; 1997. p. 19-74.

Submitted for publication June 7, 2005. Accepted July 7, 2005. 